\title{
I JORNADAS CONJUNTAS DEL COMITÉ DE GRADUADOS Y EL COMITÉ DE HIPERTENSIÓN ARTERIAL Y OTROS FACTORES DE RIESGO CARDIOVASCULAR. XVIII JORNADAS DEL COMITÉ DE GRADUADOS: "ENFERMEDAD CARDIOVASCULAR Y DIABETES"
}

\author{
I JOINT MEETING OF THE GRADUATE COMMITTEE AND \\ THE COMMITTEE ON ARTERIAL HYPERTENSION AND OTHER \\ CARDIOVASCULAR RISK FACTORS. XVIII MEETING OF THE GRADUATE \\ COMMITTEE: "CARDIOVASCULAR DISEASE AND DIABETES"
}

\author{
Comité de Graduados: \\ Coordinador: Pablo Javier Ávila \\ Secretaria: Susana Apoloni \\ Comité de Hipertensión Arterial y otros Factores de Riesgo Cardiovascular: \\ Coordinadora: Solange Houssay \\ Secretaria: Silvana Milrad
}

La enfermedad cardiovascular aterosclerótica -definida como enfermedad arterial coronaria, cerebrovascular o arterial periférica- es la principal causa de morbilidad y mortalidad para las personas con diabetes mellitus (DM). Las múltiples condiciones clínicas comórbidas que coexisten con la DM2 (por ejemplo, hipertensión arterial y dislipidemia) son factores de riesgo mayores de enfermedad cardiovascular, y la diabetes misma confiere un incremento de riesgo independiente. Varios estudios de investigación demostraron la eficacia del control de los factores de riesgo cardiovascular (FRCV) individuales en prevenir o enlentecer la enfermedad cardiovascular aterosclerótica en las personas con diabetes. Es más, se aprecian grandes beneficios cuando se controlan múltiples FRCV en forma simultánea?.

La insuficiencia cardíaca (IC) es otra causa mayor de morbilidad y mortalidad por enfermedad cardiovascular. Estudios recientes reportaron que las tasas de hospitalización por IC incidente (ajustadas por edad y sexo) fueron dos veces superiores en personas con DM que en aquellas sin DM. Los pacientes con DM padecen IC con fracción de eyección preservada (FEp) y también con fracción de eyección reducida (FEr). La hipertensión arterial (HTA) y la enfermedad arterial aterosclerótica son, con frecuencia, precursores de ambas formas de
IC, mientras que el infarto de miocardio (IM) es un factor de riesgo mayor para IC con $\mathrm{FEr}^{2}$.

Para prevenir y tratar la enfermedad cardiovascular aterosclerótica y la IC, los FRCV deben valorarse sistemáticamente al menos anualmente en todos los pacientes con DM. Estos FRCV incluyen la obesidad, el sobrepeso, la HTA, la dislipidemia, el tabaquismo, la historia familiar de enfermedad arterial coronaria prematura, la enfermedad renal crónica y la presencia de albuminuria. Los FRCV modificables deben evaluarse, tratarse y controlarse.

En los últimos años se ha confirmado que algunos fármacos para tratar la diabetes no sólo son seguros desde el punto de vista cardiovascular, sino que incluso demostraron capacidad para reducir el riesgo de enfermedad cardiovascular en la DM2. Dado que la prevalencia de enfermedad cardiovascular es cinco veces mayor en personas con DM vs la población general, resulta prioritario identificar los fármacos capaces de disminuir la mortalidad y morbilidad por enfermedad cardiovascular ${ }^{3}$.

Las Jornadas de Graduados se celebran desde hace 36 años cada dos años, teniendo como participantes a médicos egresados de la Escuela de Diabetes de la Sociedad Argentina de Diabetes (SAD), con representantes de todas las provincias de Argentina, con el fin de realizar un estudio detallado sobre un aspecto particular de la diabetes. 
El objetivo de estas Jornadas Conjuntas intercomités de Graduados y de Hipertensión Arterial y otros Factores de Riesgo Cardiovascular fue realizar una revisión bibliográfica del tema: “Enfermedad cardiovascular y diabetes." Para ello, se dividió a los 43 participantes en tres mesas de trabajo que realizaron la revisión y actualización de los siguientes temas:

- Mesa 1: Diabetes mellitus tipo 1 y enfermedad cardiovascular.

- Mesa 2: Insuficiencia cardíaca y diabetes mellitus tipo 2.

- Mesa 3: Efectos cardiovasculares de los fármacos antidiabéticos.

El 4 de octubre de 2019 se realizó la presentación del material preparado por cada integrante de cada una de las mesas, con la coordinación de un representante de cada uno de los comités participantes, junto con un panel de expertos en diabetes y en enfermedad cardiovascular.

El 5 de octubre se llevó a cabo la presentación del resumen final de los contenidos revisados por cada una de las mesas ante todos los participantes del encuentro.

Agradecemos la invaluable y generosa participación y colaboración de los miembros del Comité de Graduados de la SAD, de los miembros del Comité de Hipertensión Arterial y otros Factores de Riesgo Cardiovascular, de los coordinadores, secretarios, del panel de expertos en diabetes y del panel de expertos en cardiología, y presentamos este resumen de los temas desarrollados en las Jornadas.

\section{BIBLIOGRAFÍA}

1. Gaede P, Lund-Andersen $\mathrm{H}$, Parving $\mathrm{HH}$, Pedersen O. Effect of a multifactorial intervention on mortality in type 2 diabetes. $\mathrm{N}$ Engl J Med 2008; 358:580-591.

2. Cavender MA, Steg PG, Smith SC Jr, et al.; REACH Registry Investigators. Impact of diabetes mellitus on hospitalization for heart failure, cardiovascular events, and death: outcomes at 4 years from the Reduction of Atherothrombosis for Continued Health (REACH) registry. Circulation 2015; 132:923-931.

3. Dieuzeide G, Puchulu F, Sanabria H, Sinay I. Efectos cardiovasculares de nuevos fármacos no insulínicos en diabetes. Medicina (Buenos Aires) 2018; 78:185-193. 